\title{
PRESUPERNOVAE*
}

\author{
V. S. IMSHENNIK and D. K. NADYOZHIN \\ Institute of Applied Mathematics, USSR Academy of Sciences, Moscow, U.S.S.R.
}

\begin{abstract}
A survey is given of the present state of theories concerned with the mechanism of supernova outbursts. Special attention is paid to the state of the star immediately before the supernova explosion (presupernova).

The following topics are discussed.

(1) The thermonuclear model of supernova explosions.

(2) The gravitational collapse model of supernova explosions.

(3) The characteristics of presupernova models resulting from the comparison of theoretical light curves with observational ones.
\end{abstract}

\section{General Survey}

The problem of supernovae is an extremely interesting one from very different points of view both theoretical and observational. It is closely connected with a number of various astrophysical topics from the origin of cosmic rays and chemical elements to the origin of pulsars and black holes.

The internal structure of the star immediately before a supernova outburst is very important for elucidation of mechanisms of supernova explosions. Thus, a problem of presupernovae arises. At the present time it is indisputable that the stars explode as supernovae at the late evolutionary stages when main stores of nuclear fuel in stellar core have exhausted due to thermonuclear fusion. However, all intermediate products up to primordial hydrogen and helium at the outermost layers of a star may be in stellar envelope, provided they have not been ejected because of mass loss, or mixed with interiors.

As the theory of late stages of stellar evolution is going on, overcoming great difficulties connected with consideration of convection, mass loss, kinetics of thermonuclear reactions, multishell heterogeneous stellar structure, neutrino energy losses, and so on, we obtain a still more clear idea of presupernova structure. For the present, however, it is difficult to deduce a definite point of view. Therefore, the theory of supernovae began its own independent development based on very simple and crude presupernova models which are usually chosen from general considerations. Such a method is fully justified as it gives a chance to obtain some additional information about presupernovae by means of comparing theoretical results with observational data. It seems for us that the double approach to the problem of presupernovae, both from the side of stellar evolution and of hydrodynamical explosion theory, will finally permit us to solve the problem and, hence, to construct the full picture of star life from its birth to death. The problem of presupernovae will be discussed in this report mainly from the hydrodynamic point of view.

* This paper was presented by D K. Nadyozhin. 
Roughly speaking there is a rather trivial alternative for the present. In the first place a star may pass over all late stages of its evolution just quietly without disruption including possible stages of slow mass loss. In that case the central regions of the star burn nuclear energy supplies up to full exhaustion and the matter transmutes to the iron group elements. In the second place a star may experience the thermonuclear explosion due to carbon, carbon-oxygen or another flash. This phenomenon was first discovered as a helium flash. It is one of the greatest difficulties in the theory of stellar evolution up to now.

In the first case dynamical instability leading to gravitational collapse develops at centre of the star. The gravitational collapse is followed by powerful neutrino radiation and thermonuclear explosion of nuclear fuel remaining in the star's envelope. Various supernova models of that type have been constructed for a wide range of presupernova masses (Fowler and Hoyle, 1965; Colgate, White, 1966; Arnett, 1966, 1967; Ivanova et al., 1967, 1969; Rakavy and Shaviv, 1967; Fraley, 1968; Finzi and Wolf, 1967; Hansen and Wheeler, 1969). These models differ one from another by the mechanisms of dynamic instability and by nature of the physical conditions involved. The investigation of dynamic instability and a survey of theoretical works on such types of supernovae can be found in the book by Zel'dovich and Novikov (1971). In these works, largely using numerical solutions of hydrodynamic equations, there is a difficulty which consists in the low efficiency of adopted physical mechanisms of ejection of a star's envelope such as thermonuclear explosion of remaining nuclear fuel and neutrino energy deposition. However, the difficulty doesn't concern presupernovae with very large masses $\left(M \geqslant 30 M_{\odot}\right)$ and sufficiently low ones $(M \approx$ $\left.\approx M_{\text {Chandr }}=1.4 M_{\odot}\right)$, (Fraley, 1968; Hansen and Wheeler, 1969). These presupernovae probably don't reach the evolutionary stage with an iron core. The very massive stars are dynamically unstable due to electron-positron pair creation followed by thermonuclear explosion of a carbon-oxygen core (Fraley, 1968; Rakavy and Shaviv, 1967). Dynamic instability due to neutronization developing on the time-scale of betaprocesses (Bisnovatyi-Kogan and Kazhdan, 1966; Bisnovatyi-Kogan and Seidov, 1969, 1970 ; Imshennik and Chechetkin, 1970) is the cause of explosion of the stars with masses near Chandrasekhar's limit. The iron core must be formed as result of evolution of the intermediate-mass stars. The main mechanism of dynamic instability in this case is disintegration of the iron group elements to $\alpha$-particles and free nucleons (Fowler and Hoyle, 1965). As gravitational collapse proceeds, the central regions of the star become opaque first to the electron neutrino and then to muon neutrino (Domogatsky, 1969). At the moment of ignition of the nuclear fuel remaining in the stellar envelope, the central regions of the star form a small-size core opaque to neutrino radiation characterized by the following parameters: $T_{c}=100-300 \times 10^{9} \mathrm{~K}, \varrho_{c}=10^{12}-10^{14} \mathrm{~g} \mathrm{~cm}^{-3}$, $R \approx 10^{7} \mathrm{~cm}$. The interior of the star having dimensions from $10^{7}$ to $10^{9} \mathrm{~cm}$ absorbs diluted radiation of the neutrino photosphere and simultaneously loses the energy because of volume radiation. That model seems the most plausible one for the problem of heating external layers by neutrino radiation (deposition). In the first works involving the effect of deposition, the deposition power apparently was strongly over- 
estimated (Colgate and White, 1966; Arnett, 1966). Ivanova et al. (1967) found more moderate estimation of deposition power and in addition took into consideration the kinetics of oxygen burning. The total energy of a supernova explosion found in that work proved to be of the order of $3 \times 10^{50} \mathrm{erg}$. It is quite enough to explain observational data (Poveda and Woltjer, 1968). To answer a question about the role of deposition it is necessary to solve a problem characterized by consideration of energy transport by the way of neutrino heat conductivity in a stellar core opaque to neutrino radiation (Imshennik and Nadyozhin, 1972), and in a time allowing for kinetics of $\beta$-processes in the field of diluted neutrino radiation in outer stellar layers.

It should be noted that a star's rotation and magnetic field favouring ejection of the envelope have not yet been taken into account appropriately. The investigations in that direction involving great difficulties have been started quite recently (BisnovatyiKogan, 1970; LeBlanc and Wilson, 1970).

In the alternative discussed above, there was mentioned the second possibility of supernova outburst as a result of catastrophic development of carbon or, in evolutionary sense, more late flash. The idea of a thermonuclear explosion in degenerate matter was known long ago. However, only in 1969 was the intensive theoretical elaboration of that version of supernova started (Arnett, 1969). At present time a great number of works are devoted to the problem (Colgate, 1971; Barkat et al., 1970, 1971; Buchler et al., 1971; Bruenn, 1971, 1972). In the first work by Arnett (1969) a dense $\left(\varrho_{c} \gtrsim 2 \times\right.$ $\times 10^{9} \mathrm{~g} \mathrm{~cm}^{-3}$ ) degenerate carbon core has been shown to suffer first a thermal instability and then to explode in regime of detonation. In contrast with previously adopted presupernovae for which initial models had been generally constructed rather arbitrarily (without necessary connection with the late stages of stellar evolution), Arnett (1969) has pointed out appropriate evolutionary models $\left(3.5 M_{\odot}<\right.$ $<M<8 M_{\odot}$ ) characterized by a natural origin of a degenerate carbon-oxygen core. The models have in more detail been calculated also by Rose (1969), Paczyński (1970), and Uus (1970). It is impossible, however, to say that a full fitting of the calculations of stellar evolution with the initial state of stellar core before explosion is now available. Therefore, there is rather great arbitrariness of the choice of the initial presupernova models connected with the mass exchange in binary stars (mass loss for a single star), the neutrino energy losses, the nuclear reaction rate etc. (Bruenn, 1972). The most complete hydrodynamic calculations of the thermonuclear explosion of the stellar core have been made by Bruenn (1971, 1972). These works, as distinct from previous analysis by Arnett (1969), involve the following complex physical processes which relax the detonation: (1) the decomposition of iron group elements at high temperatures $\left(T \gtrsim 10^{10} \mathrm{~K}\right)$ produced both inside and behind the detonation front, (2) various $\beta$-processes such as URCA-process followed by the powerful neutrino radiation and neutronization. As compared with Arnett's work in which full expansion of star with total energy $2 \times 10^{51} \mathrm{erg}$ had been obtained, consideration of the relaxation effects mentioned above has resulted in the possibility of formation of a gravitationally bound remnant and even a neutron star.

Now is the time to say that by contrast with dynamically unstable presupernovae 
(without regard to stars with extreme masses), it is very difficult to find a gravitationally bound remnant in the case of thermonuclear presupernovae. In this respect the theory of supernovae perhaps fatally contradicts the observations confidently indicative of the relation of pulsars or rotating neutron stars with the remnants of supernova explosions (Zel'dovich and Novikov, 1971). Bruenn's calculations resulted in the formation of a gravitationally bound remnant for central densities $\varrho_{c} \gtrsim 1.5 \times$ $\times 10^{10} \mathrm{~g} \mathrm{~cm}^{-3}$. From the standpoint of stellar evolution theory $\left(T_{c} \sim \varrho_{c}^{1 / 3}\right)$, in that case the central region of the star most likely has already passed over the stage of carbon burning and is composed of its products: $\mathrm{O}, \mathrm{Ne}, \mathrm{Mg}, \ldots$. The analysis of selfconsistency of the detonation (with allowance for nonideality of ion component) has led to doubtful results namely for a such mixture (Bruenn, 1972). Therefore, in spite of a gravitationally bound remnant having been obtained in hydrodynamic calculations, we have to recognize that it was formed under very trying conditions. In short, the neutron stars indeed hardly form in the supernova theory based on a flash of nuclear fuel in a dense stellar core. Certain new possibilities in this respect seem to arise from the really existing pulsational regime of thermonuclear explosion which has been found as result of nuclear kinetics being taken into account in hydrodynamic calculations (Ivanova et al., 1973). The previous idea of the way of detonation development proved to be inexact. The central regions of the star burn without forming a detonation front and the star as a whole suffers radial pulsations. At the contraction phases the portions of matter are burnt out with gradually increasing pulsational amplitude due to resulting energy release. This is the way to obtain mass ejection at one of expansion phases followed by burning extinction and the subsequent formation of a gravitationally bound remnant. Decomposition of iron group elements and $\beta$-processes, unfortunately not allowed for the calculations, favour the conclusion. The above calculations resulted in full destruction of the star after the detonation front had been formed in the third burning cycle (the third contraction) at $m / M \approx 0.5$. A remaining half nuclear fuel burnt out in the regime of detonation. From the analysis of results it follows, however, that precise values of the initial data (especially temperature distribution) are very significant.

Finally, another difficulty should be mentioned. There is a doubt as to the fact of thermonuclear explosion due to carbon burning. Paczyński (1972) assumed that the neutrino losses by URCA-processes proceeding in a convective core may stabilize carbon burning. This is a generalization of URCA-shell mechanism taking place in the case of white dwarf vibrations (Tsuruta and Cameron, 1970). Although the first calculations of selfconsistent carbon-oxygen models allowing for URCA-losses have not led to sufficiently large burning relaxation (Ergma and Paczyński, 1973), the effect does change our notion of the conditions of the thermonuclear explosion. In this respect it is necessary to point to the calculations of a carbon-oxygen star's evolution for a large range of stellar masses $1.5 \leqslant M / M_{\odot} \leqslant 30$ (Ikeuchi et al., 1971, 1972). The calculations resulted in passing through the stages of carbon and later fuels burning well, without appreciable development of thermal instabilities. The dynamically unstable presupernovae with different masses of iron core (in dependence on initial 
stellar mass) have been obtained in the calculations by the evolutionary method.

When investigating supernova dynamics and also late stages of stellar evolution one often considers only a stellar core neglecting the extensive envelope. It is, of course, reasonable since in the absence of mixing a rarefied envelope has essentially no influence on processes proceeding in the core. The theory, however, must answer a basic question: what will happen to a star with a mass $M_{m s}$ on the main sequence? In this connection it is useful to give Table I (all masses are given in solar units).

TABLE I

\begin{tabular}{rcccc}
\hline$M_{m s}$ & $M_{\text {conv }}$ & $M_{\text {CO }}$ & $M_{\text {Femin }}$ & $M_{\text {Fei }}$ \\
\hline 64 & 48 & 26 & 2.8 & 26 \\
32 & 19 & 10 & 2 & 10 \\
16 & 6.8 & 3 & 1.5 & 3 \\
8 & 2.4 & $\sim 1.4$ & $\sim 1$ & $\sim 1.4$ \\
4 & 0.8 & $\sim 1.4$ & $\sim 1$ & $\sim 1.4$ \\
\hline
\end{tabular}

Here $M_{m s}$ denotes the mass of the star at main sequence; $M_{\text {conv }}$, the mass of the convective core at main sequence; $M_{\mathrm{Co}}$, the mass of carbon-oxygen core; $M_{\mathrm{Femin}}$, the mass of iron core in absence of mixing due to circulation currents; $M_{\mathrm{Fei}}=M_{\mathrm{CO}}$, the mass of iron core in the case of carbon-oxygen core evolving with full mixing. The table was made up in accordance with Varshavsky and Tutukov $(1972,1973)$, Ikeuchi et al. (1971, 1972), and Paczyński (1971). As appears from the table the iron cores of massive stars $\left(M_{m s} \gtrsim 4 M_{\odot}\right)$ finishing their evolution are of the order of 1-3 $M_{\odot}$. One must have in view, however, that for the present time the role of circulation (and partially convective) mixing is not understood in the theory of advanced stellar evolution. With a sufficiently effective mixing the masses of iron cores may be as high as $M_{\mathrm{Fei}}$.

At this time there are two basic ideas of the presupernova structure. Firstly, there is a star having fully exhausted nuclear fuel in its central region and being just on the boundary of dynamical instability. Secondly, there is a star with the degenerate dense core consisting of carbon or other later nuclear fuels immediately before ignition. Thermal and vibrational instabilities are of importance here.

A lot of information about presupernovae can be obtained by comparing theoretical calculations with observational data on the light curves, the velocities of ejected matter etc. A number of works (Imshennik and Nadyozhin, 1964, 1967; Grassberg and Nadyozhin, 1969a, b; Grassberg et al., 1971) deal with the ejection of supernova envelope by shock waves and the construction of theoretical light curves. The fundamental idea of these works consists in a supernova light curve being a result of illumination of thermal and recombination energy kept in outer layers of the star which have been heated by shock and (or) thermal wave. If a supernova has a compact (homogeneous) structure, almost all shock energy is converted into kinetic energy of envelope expansion and only a small part of the order of $10^{-3}-10^{-4}$ is lost in the form of radiation. If the dense core of a presupernova is surrounded by a rarefied extended envelope, 
the process proceeds in a quite different way: the powerful thermal wave is generated in front of the shock propagating in stellar envelope. The time scale of thermal relaxation of the external layers proves to be less than a hydrodynamic one. Therefore, the part of energy lost in form of radiation increases considerably up to $0.02-0.05$ for the presupernovae with the dimensions of envelope of the order of $10^{4} R_{\odot}$.

The presupernovae models with the extended envelopes explain the basic observational data of light curves fairly well. The compact models are in a good agreement with anomalous light curves of such supernovae as in NGC 5457. Thus there is a principal possibility to find out from observational data whether a presupernova was compact or whether it had an extensive envelope. A peculiar cooling regime of expanding supernova envelopes in the form of cooling and recombination wave was found out in the calculations. In extended models this regime takes place in a few weeks after light curve maximum and may be responsible for the 'hump' observed on the light curves of supernova of type II. In compact models the cooling wave explains almost the whole of observed light curve except a sharp peak of radiation resulting from the emergence of the shock wave. For an explanation of the light curves of supernovae of type II it is necessary to use the presupernova models with very extended envelopes $\left(\sim 10^{4} R_{\odot}\right)$. In that case it is sufficient that the envelope mass should be only $0.1-1 M_{\odot}$. It must be emphasized that the envelope may not at all be in hydrostatic equilibrium. For example, it may be in the state of ejection with velocities of the order of a few hundreds $\mathrm{km} \mathrm{s}^{-1}$. The above thermal theory of light curves refers basically to supernovae of type II.

In the case of supernovae of type I nonequilibrium processes and also some additional energy sources such as $\beta$-decay may be of great importance. The problem was thoroughly discussed by Colgate and McKee (1969). They found that the postmaximum part of the light curve may be explained by two-step decay of radioactive nuclei ${ }^{56} \mathrm{Ni}\left({ }^{56} \mathrm{Ni} \rightarrow{ }^{56} \mathrm{Co} \rightarrow{ }^{56} \mathrm{Fe}\right)$. It is necessary that about $0.3 M_{\odot}$ of ${ }^{56} \mathrm{Ni}$ should be in stellar envelope. Radioactive theory is in a good agreement with the exponential part of the light curve and with a lack of ultraviolet radiation (near the maximum of light the theoretical effective temperature equals $\sim 6500 \mathrm{~K}$ ). Though the fluorescence effects may be of some importance for supernovae of type I, an attempt to explain light curves by fluorescence only (Morrison and Sartori, 1969) is connected with great difficulties (Grassberg et al., 1971).

The lack of equilibrium between radiation and matter may result from the dominating contribution of Compton scattering to opacity because of energy exchange between photons and electrons being very small. In that case the propagation of radiation in a moving medium should be considered on the basis of equation of radiative transfer with the motion of matter taken into account (Imshennik and Morosov, 1969).

A lot of information can be in principle derived from the spectra of supernovae. The data on composition of supernova envelopes are particularly important. For example, an almost complete lack of hydrogen in external presupernova layers follows from the interpretation of supernova spectra of type I suggested by Mustel (1971). This fact 
may result either from the star having lost the hydrogen-helium envelope during its previous evolution or evolving with full mixing since some time.

\section{The Computations of Supernova Outbursts and Gravitational Collapse}

In this section of the report we shall discuss the results of the calculations of the instabilities developing in presupernovae. It is quite clear, that the analysis of the results and its comparison with observational data give us another opportunity for choosing more real presupernova models, besides its deriving by direct computation from the late evolutionary stages. We shall give below some details of our calculations concerning the problems discussed above: (1) the catastrophic development of a carbon flash followed by thermonuclear explosion in a dense carbon core, (2) the gravitational collapse of a completely evolved stellar core followed by explosion of nuclear fuel remaining in the envelope, (3) propagation of thermal and shock waves through a presupernova envelope, the dynamics of envelope expansion, and the supernova light curve.

\subsection{THERMONUCLEAR EXPLOSION OF DENSE CARBON CORE}

In contrast with previous works we have included the kinetics of thermonuclear reaction. The hydrostatic equilibrium of an initial configuration with mass $1.4 M_{\odot}$ was obtained by the relaxation method. The adopted equation of state is characterized by allowing both for degeneracy and relativity of electrons and positrons and also by taking ions in the approximation of perfect gas. For the sake of simplicity it was assumed that stellar core was composed of pure carbon. The rate of the nuclear reaction ${ }^{12} \mathrm{C}+{ }^{12} \mathrm{C} \rightarrow{ }^{24} \mathrm{Mg}+\gamma$ was taken into consideration. The initial data for hydrodynamic calculations are given in Figure 1. The initial temperature distribution is approximately indicated by solid line $\left(T_{9}=0.8\right.$ quite near the centre). The distribution imitates the thermal stage of a carbon flash which is very long in comparison with hydrodynamic time scale $\left(\sim 2 \times 10^{5} \mathrm{~s}\right.$ against $\sim 2 \mathrm{~s}$ respectively $)$. The equations of hydrodynamics in Lagrangian form

$$
\begin{aligned}
& \frac{\partial r}{\partial t}=u \\
& \frac{\partial u}{\partial t}=-4 \pi r^{2} \frac{\partial P}{\partial m}-\frac{G m}{r^{2}} \\
& \frac{\partial E}{\partial t}+P \frac{\partial}{\partial t}\left(\frac{1}{\varrho}\right)=\varepsilon_{\text {nuc }}-\varepsilon_{v} \\
& \frac{\partial r^{3}}{\partial m}=\frac{3}{4 \pi \varrho}
\end{aligned}
$$

were solved numerically together with the equation of carbon burning

$$
\frac{\partial X_{c}}{\partial t}=-f\left(\varrho, T, X_{c}\right) \text {. }
$$




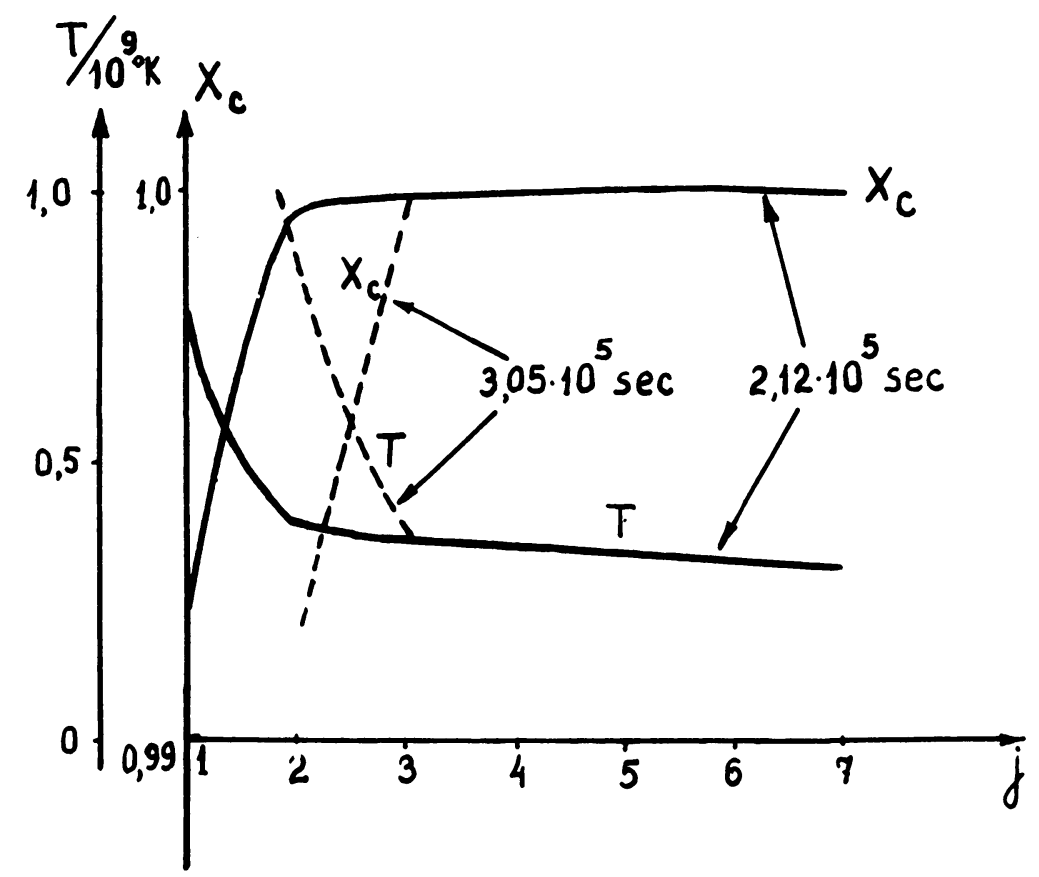

Fig. 1. Temperature and concentration of carbon before the beginning of calculations as the functions of Lagrangian coordinate $j$ (the mass scale is indicated in Figure 2).

In (1)-(5) $r$ denotes the radius; $\varrho$, the density; $T$, the temperature; $P$, the pressure; $u$, the velocity; $E$, the specific energy; and $X_{c}$, the concentration of carbon by weight. In the approximation of instantaneous conversion of ${ }^{24} \mathrm{Mg}$ to ${ }^{56} \mathrm{Ni}$ one may derive the following relation for nuclear energy generation

$$
\varepsilon_{\text {nuc }}=7.64 \times 10^{17} f\left(\varrho, T, X_{c}\right) \operatorname{erg~g}^{-1} \mathrm{~s}^{-1} .
$$

The function $f\left(\varrho, T, X_{c}\right)$ with corrections due to screening and ${ }^{24} \mathrm{Mg}$ burning up to ${ }^{56} \mathrm{Ni}$ was taken according Fowler and Hoyle (1965):

$$
\begin{aligned}
\log f\left(\varrho, T, X_{c}\right)= & 27.00+\log \left(\varrho X_{c}^{2}\right)-\frac{2}{3} \log T_{9}+ \\
& -\frac{36.57}{T_{9}^{1 / 3}}\left(1+0.08 T_{9}\right)^{1 / 3}+1.65 \varrho_{9}^{1 / 3} / T_{9} .
\end{aligned}
$$

Only URCA-proceses were not included in the neutrino losses $\varepsilon_{v}$. Figures 2-6 show the results of the calculations. The distributions of $T, \varrho, P$, and $u$ (Figures 2-5) are presented for a set of times given in Table II.

Figure 6 shows the variation of integral energies:

$$
\mathscr{E}_{\mathrm{in}}=\int_{0}^{M} E \mathrm{~d} m, \quad \mathscr{E}_{g}=-\int_{0}^{M} r \frac{G m}{r} \mathrm{~d} m, \quad \mathscr{E}_{k}=\int_{0}^{M} \frac{u^{2}}{2} \mathrm{~d} m
$$




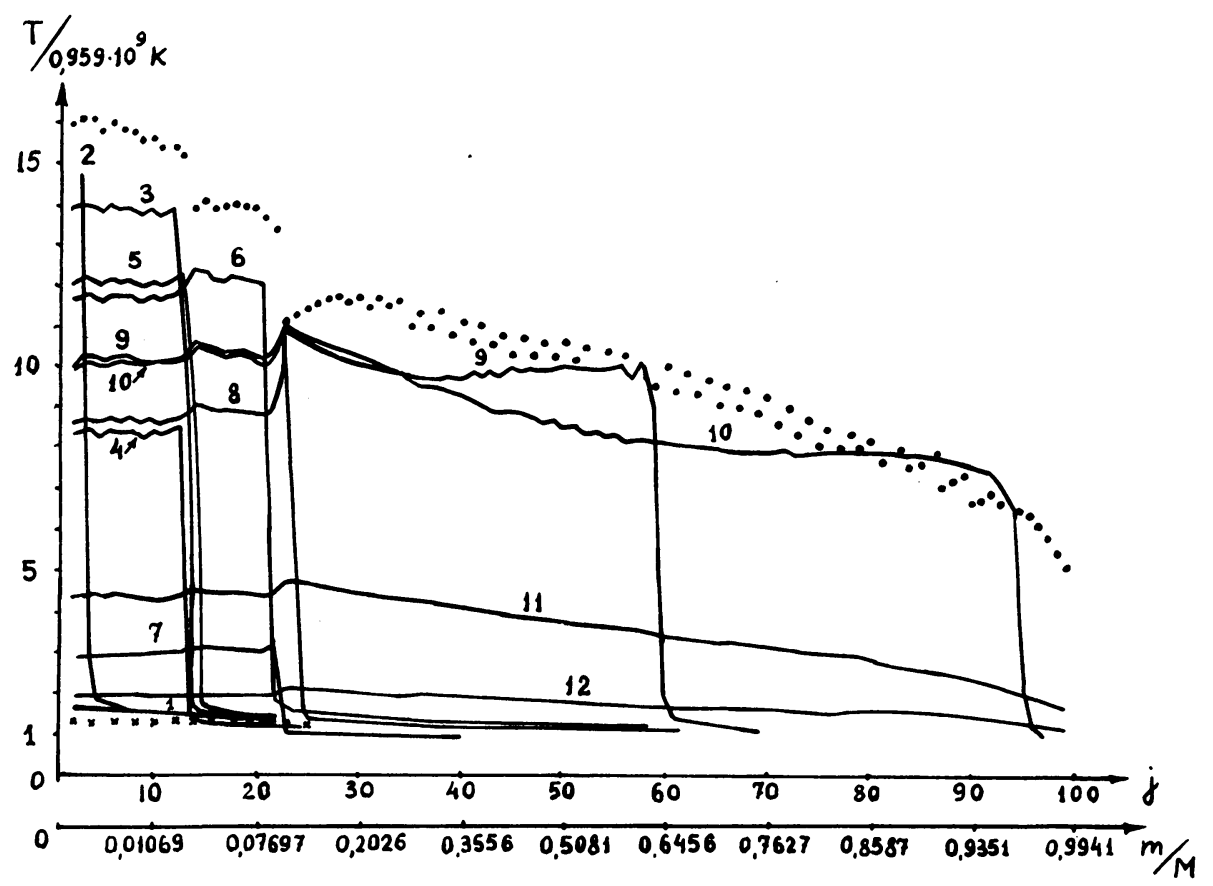

Fig. 2. Temperature as function of $m$ and $j$ for various moments of time. The circles indicate the absolute temperature maxima for every zone. The crosses correspond to temperature distribution in hydrostatically equilibrated configuration.

with time. The intervals of time corresponding to carbon burning are underlined on the axis of abscissas. The basic result of the calculations consists in finding the pulsational regime of nuclear burning. The burning first begins at the centre and gives rise

TABLE II

\begin{tabular}{|c|c|c|}
\hline $\begin{array}{l}\text { The numbers } \\
\text { in the figures }\end{array}$ & $\begin{array}{l}\text { The moments } \\
\text { of time (s) }\end{array}$ & Comments \\
\hline 1 & 10.14 & $\begin{array}{l}\text { The end of relaxation. The configuration in hydrostatic equi- } \\
\text { librium is found. }\end{array}$ \\
\hline 2 & 10.18 & The beginning of the first burning phase. \\
\hline 3 & 10.40 & The end of the first burning phase. \\
\hline 4 & 11.65 & The maximum of the first expansion. \\
\hline 5 & 12.57 & The beginning of the second burning phase. \\
\hline 6 & 12.78 & The end of the second burning phase. \\
\hline 7 & 15.42 & The maximum of the second expansion. \\
\hline 8 & 17.90 & The beginning of the third burning phase. \\
\hline 9 & 17.96 & $\begin{array}{l}\text { The beginning of the detonation. The detonation front is in } \\
\text { point } j=59 \text {. }\end{array}$ \\
\hline 10 & 18.01 & The detonation front is in point $j=94$. \\
\hline 11 & 18.34 & The beginning of total expansion. \\
\hline 12 & 18.98 & The star is in the state of total expansion. \\
\hline
\end{tabular}




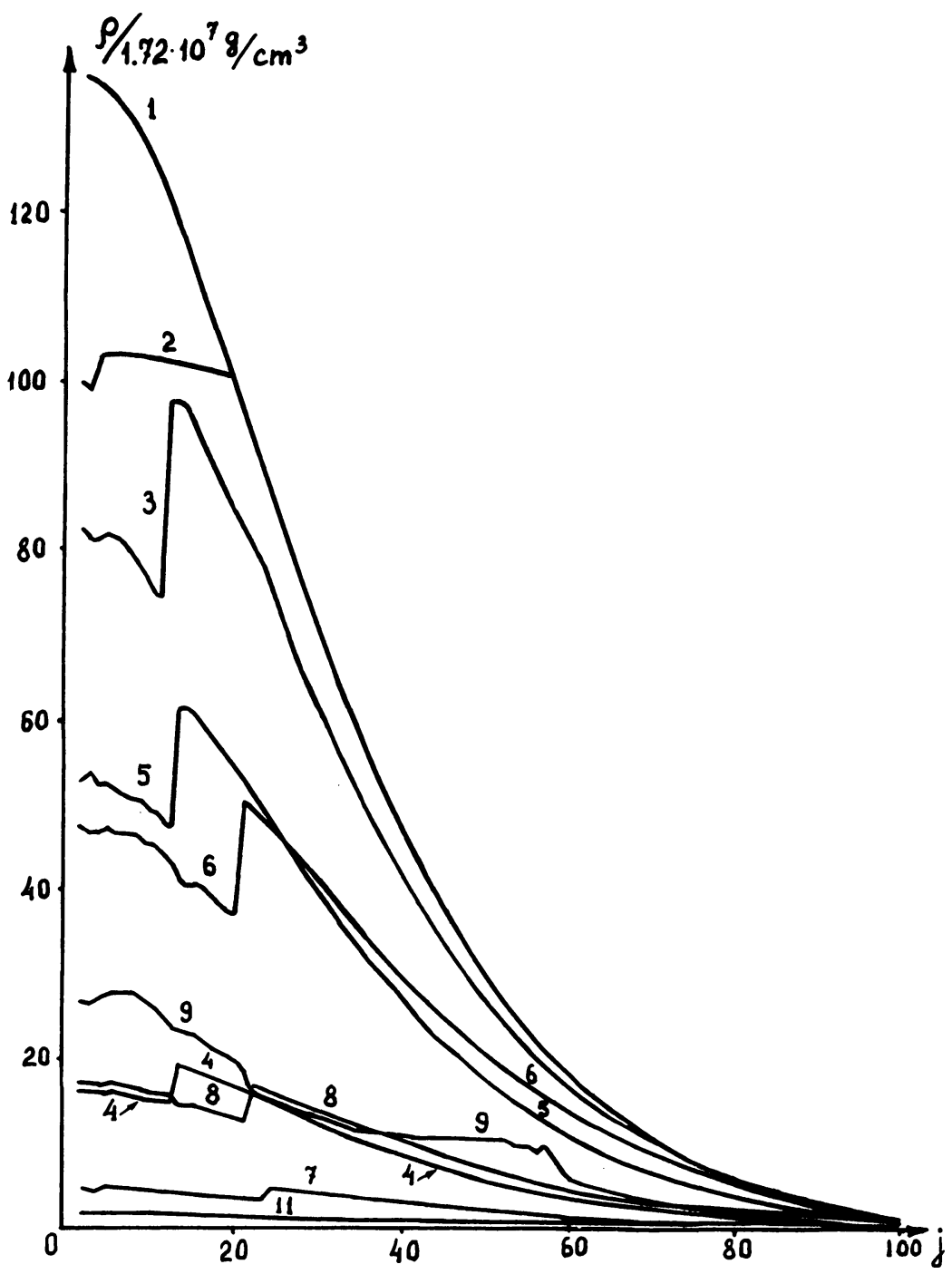

Fig. 3. Density as function of $j$ for various moments of time. The moment $t_{10}$ is excluded for technical reasons. For $t_{12}$ the density practically goes together with the axis of abscissas.

to expansion of stellar matter which in turn extinguishes the burning itself. As distinct from detonation we obviously deal with tearing the motion of matter away the zone of burning. The burning recommences in the phases of contraction of the star and then extinguishes again with following expansions. Three stages of burning were found in the calculations. The detonative burning appeared only in the third contraction on a level with relative mass $m / M \approx 0.5$. As a result the total expansion of the star occurred with complete carbon burning. However, from analysis of the calculations one may conclude that the pulsational regime of thermonuclear explosion may result 


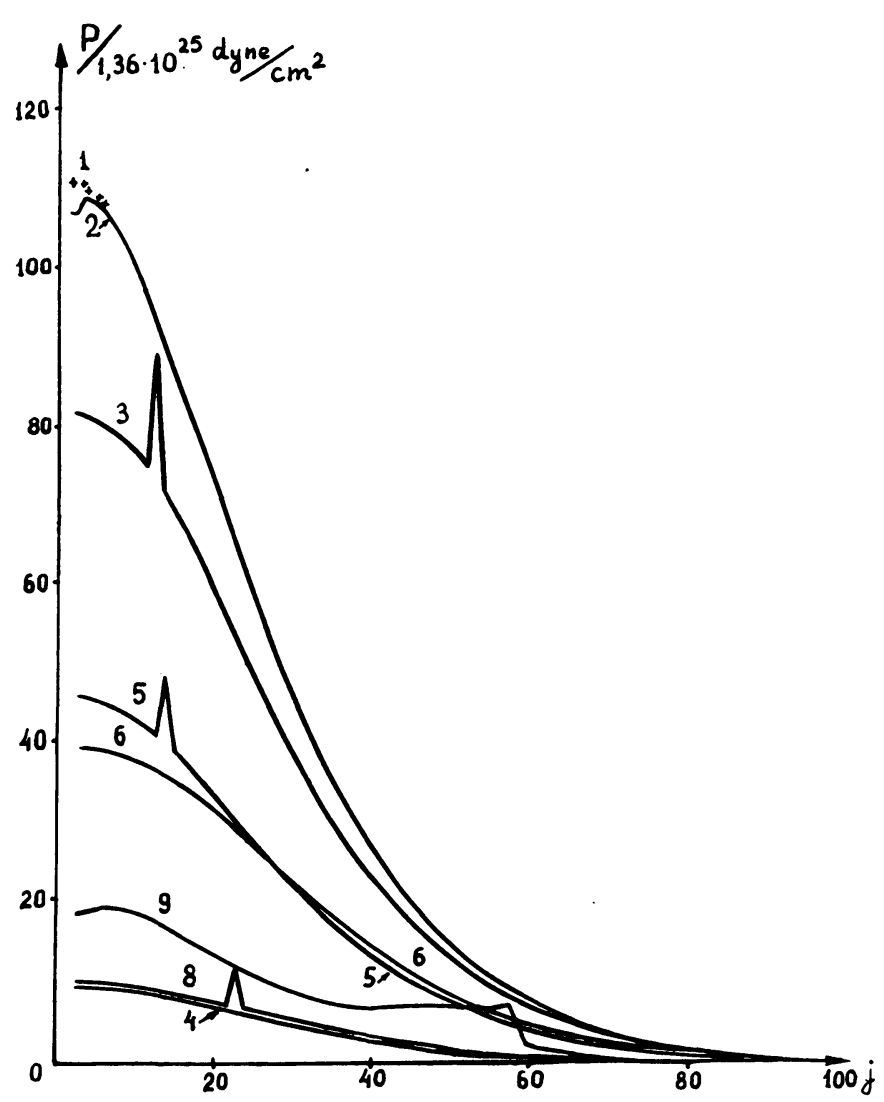

Fig. 4. Pressure for the same moments as in Figures 2 and 3.

in principally new inferences. In particular, it may facilitate the formation of a gravitationally bound remnant. Another version of the calculations with $\delta$-shape initial temperature distribution which fits thermal theory of the flash better (Figure 1) resulted in numerous pulsations without any appreciable carbon burning. In that case there would be needed a lot of pulsations in order that carbon should burn completely. Apart from great sensitivity to initial conditions in the case of pulsations, the calculations point to the importance of some physical processes such as: (1) decomposition of iron group elements to nucleons and more light nuclei, (2) $\beta$-processes, neutronization of matter, and URCA-losses with due regard of pulsational intensification of the latter. The next series of our calculations devoted to consideration of the above effects is under way.

\subsection{Gravitational collapse}

At initial stages the gravitational collapse of a stellar core devoid of nuclear fuel may be considered without recourse to GTR-effects. The Equations (1)-(6) should be used in the case. In the stellar core one must put $\varepsilon_{\text {nuc }}=0$, but in the envelope one should 


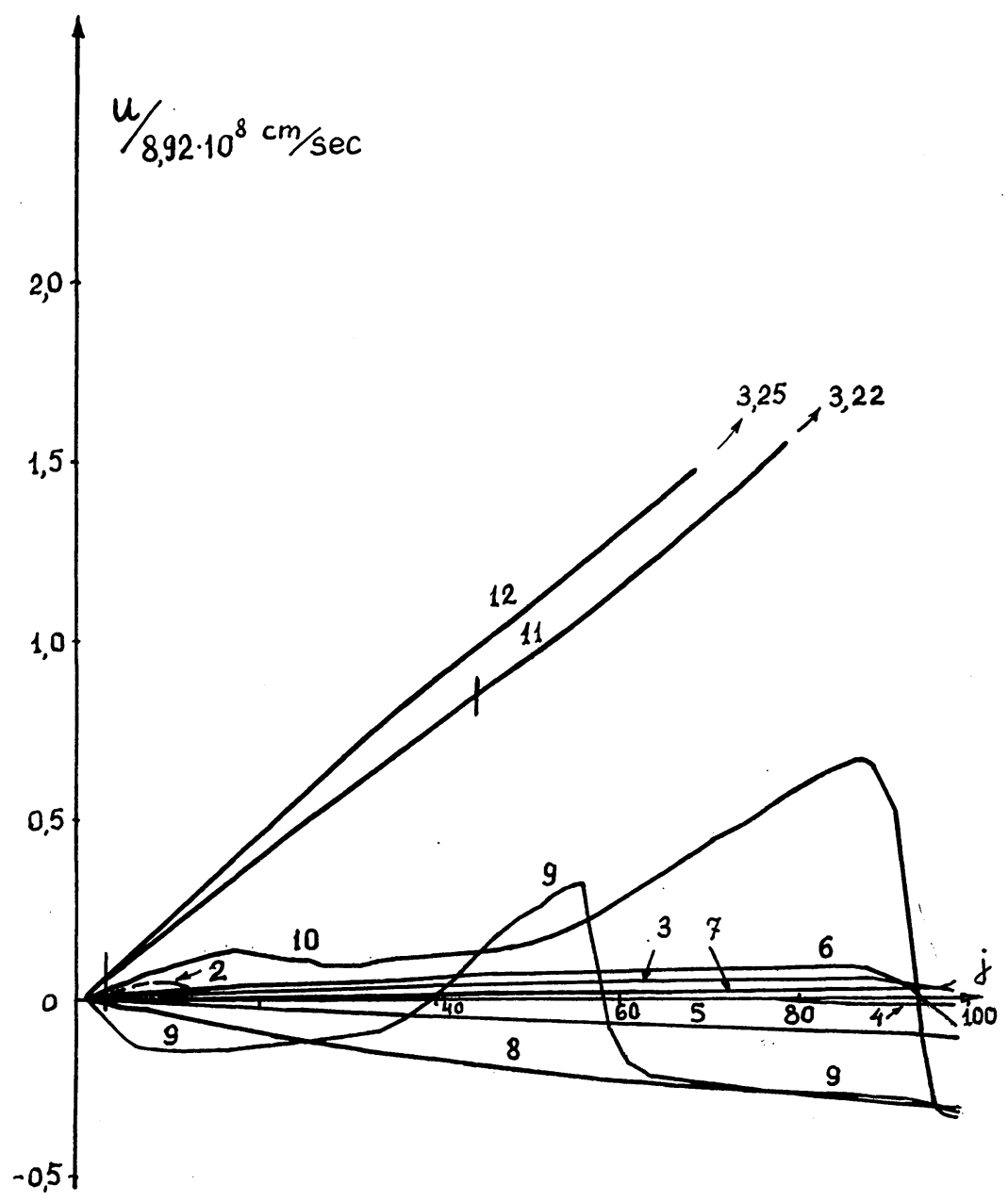

Fig. 5. Velocity for various moments of time. In moment $t_{1}$ the velocity equals to zero.

use $\varepsilon_{\text {nuc }}$ corresponding to unburned matter, which is most likely composed of $\alpha$-nuclei: ${ }^{12} \mathrm{C},{ }^{16} \mathrm{O},{ }^{24} \mathrm{Mg}, \ldots$. It is necessary to use the complex equation of state taking account of the decomposition of iron group elements to free nucleons (Imshennik and Nadyozhin, 1965; Chechetkin, 1969; Imshennik and Chechetkin, 1970). The violent reduction of the adiabatic index due to decomposition of iron is the reason of dynamic instability transiting to gravitational collapse.

Ivanova et al. $(1967,1969)$ have pointed out that, immediately after decomposition of iron, neutrino emission was due mainly to an URCA-process on free nucleons, while at the moment of stability loss neutrino emission was due largely to processes of electron-neutrino interaction (pair annıhilation, plasmon neutrino, photo-neutrino). In these works the formulae for neutrino emission due to URCA process were also derived. 


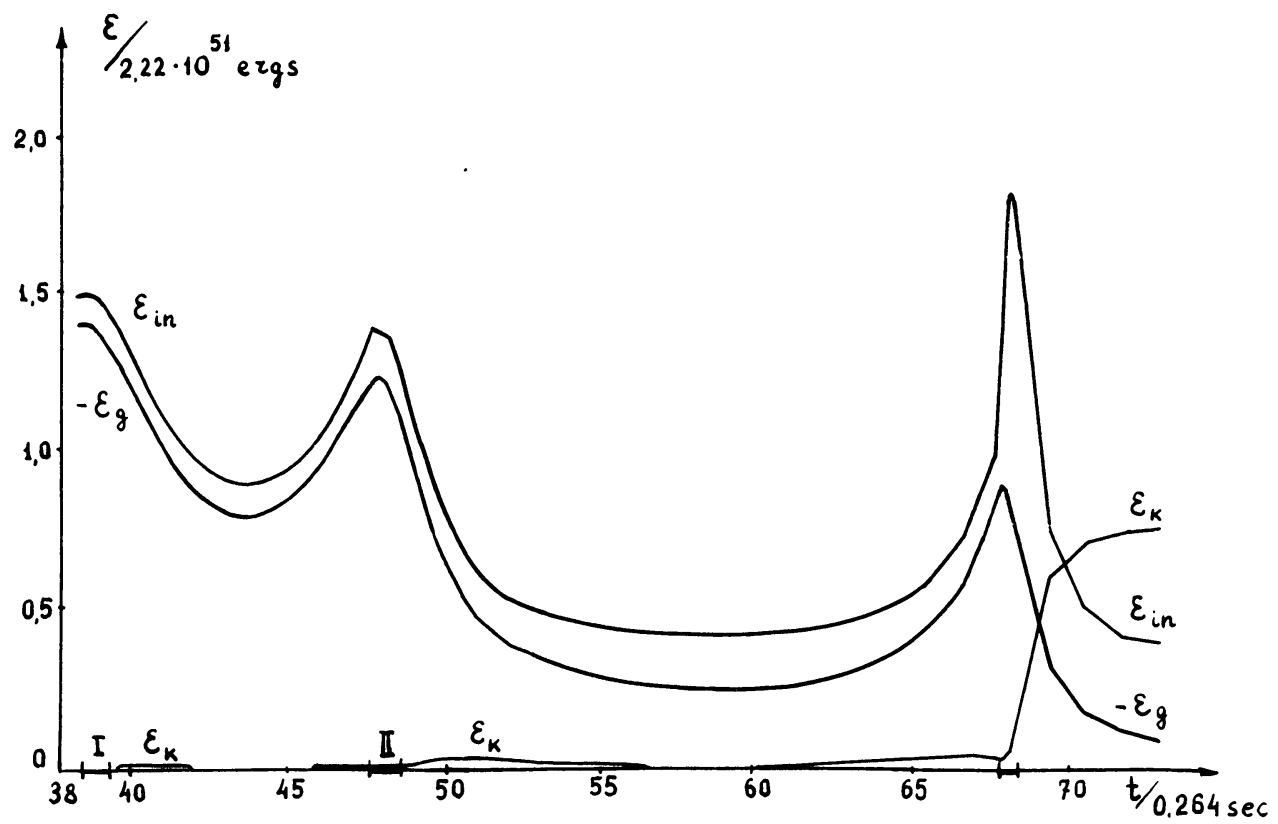

Fig. 6. Dependences of total energies $\left(\varepsilon_{k}-\right.$ kinetic energy; $\varepsilon_{\text {in }}$-- internal energy with electronpositron rest energies included; $\varepsilon_{g}$ - gravitational energy) on time. Roman numerals are used for denoting of carbon-burning at phases of contraction.

The electron neutrino 'optical' thickness of the star's core proves to be of the order of 1 when in process of the collapse central temperature and density increase up to $40 \times 10^{9} \mathrm{~K}$ and $10^{11}-10^{12} \mathrm{~g} \mathrm{~cm}^{-3}$ respectively. Therefore the processes of electron neutrino absorption are of a great importance at that stage. The absorption of neutrinos may be taken into account by multiplying the rate of volume energy radiation $\varepsilon_{v}$ at every point of the star by some factor $K$, which in very crude approximation may be used in the following form:

$$
K=e^{-\tau_{v}(r)},
$$

where $\tau_{v}(r)$ is neutrino optical depth at the distance $r$ from the centre of the star. It is connected with mean free path of neutrinos $l_{v}$ by the next relation:

$$
\tau_{v}(r)=\int_{r}^{R} \frac{\mathrm{d} r}{l_{v}} .
$$

Such a method doesn't allow for redistribution of energy transported by neutrino radiation (or deposition) which happens by the way of diffusion in the case of $\tau_{v} \gtrsim 1$. Somewhat different method was used by Ivanova et al. (1969). An analytical formula for $K$ in the star's centre was found from the exact solution of the equation of the neutrino transfer with the aid of quadratures. The formula gives the same order of $K$ 
as follows from (9) with $r=0$. It was then assumed that $K$ is constant all over the star. In the terms of (9) and (10) it was assumed that

$$
K=e^{-\tau_{v}(0)} \text {. }
$$

So the deposition was in a sense taken into consideration. A special calculation of gravitational collapse with $K$ taken from (9) and (10) was carried out to illustrate the above method. The full lines (Figure 7) indicate the dependence of $\tau_{v}(r)$ and $\varepsilon_{v} e^{-\tau_{v}(r)}$ at some moment of collapse. The dotted line was used for dependence $\varepsilon_{v} e^{-\tau_{v}(0)}$ which on the order of magnitude corresponds approximately to the work by Ivanova et al. (1969). The deficiency of neutrino radiation presented by shading (Figure 7) may be regarded as some kind of consideration of the deposition.

The results of the work in question point out that the deposition itself doesn't lead to sufficiently intensive energy transfer. However, it favours the detonation of nuclear fuel (oxygen) remaining in the stellar envelope. Energy release due to the detonation is quite sufficient for the explanation of the supernova outburst.

In collapsing supernova models it is very easy, as compared with thermonuclear models, to obtain a gravitationally bound remnant. In the course of subsequent evolution it may change into pulsar or black hole depending on its mass. Figure 8 taken from Ivanova et al. (1969) shows the distributions of velocity in Lagrangian coordinates for

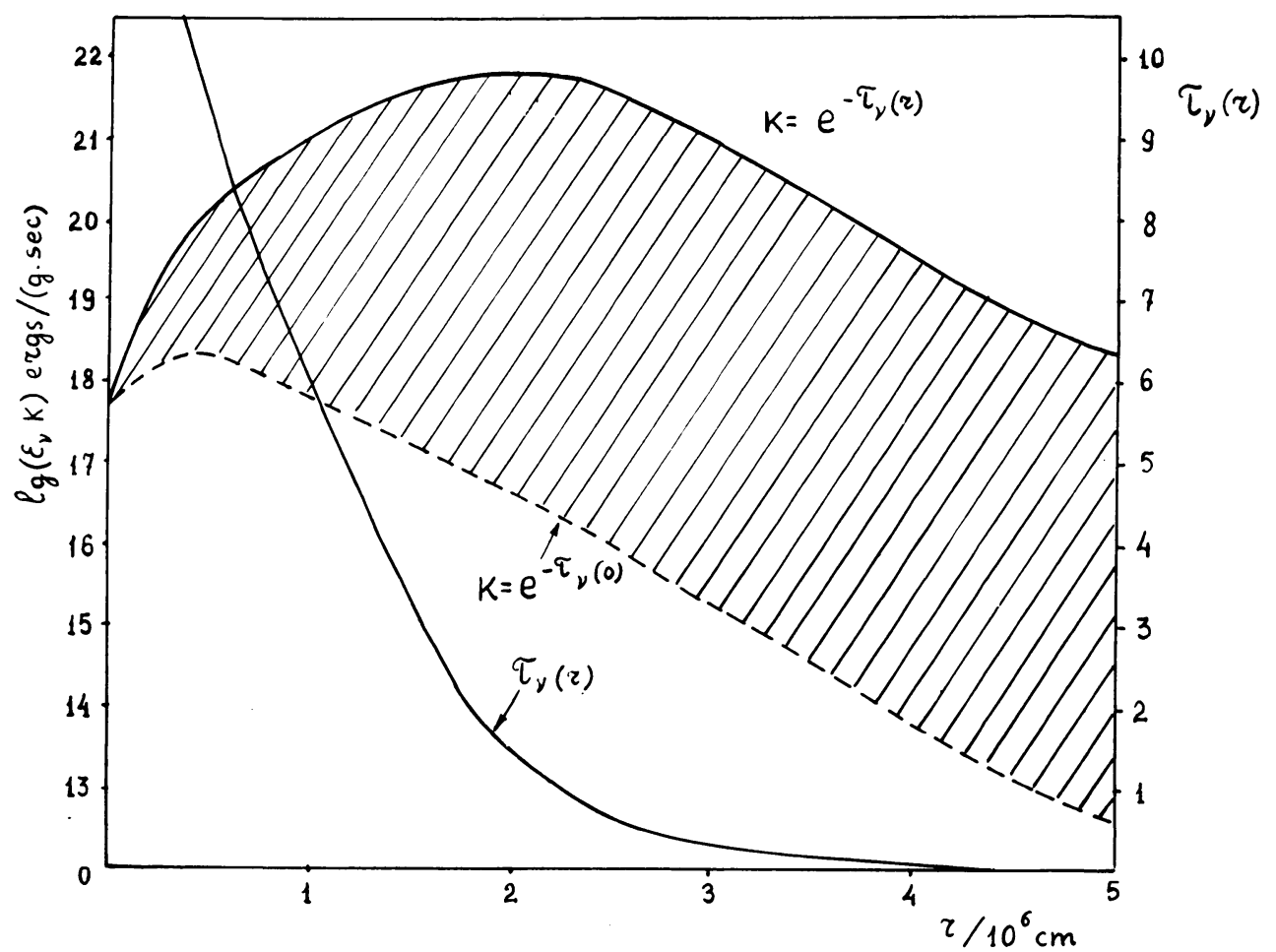

Fig. 7. Optical neutrino depth and neutrino energy losses for the various methods of considering neutrino absorption as function of Eulerian coordinate at some moment of gravitational collapse. 


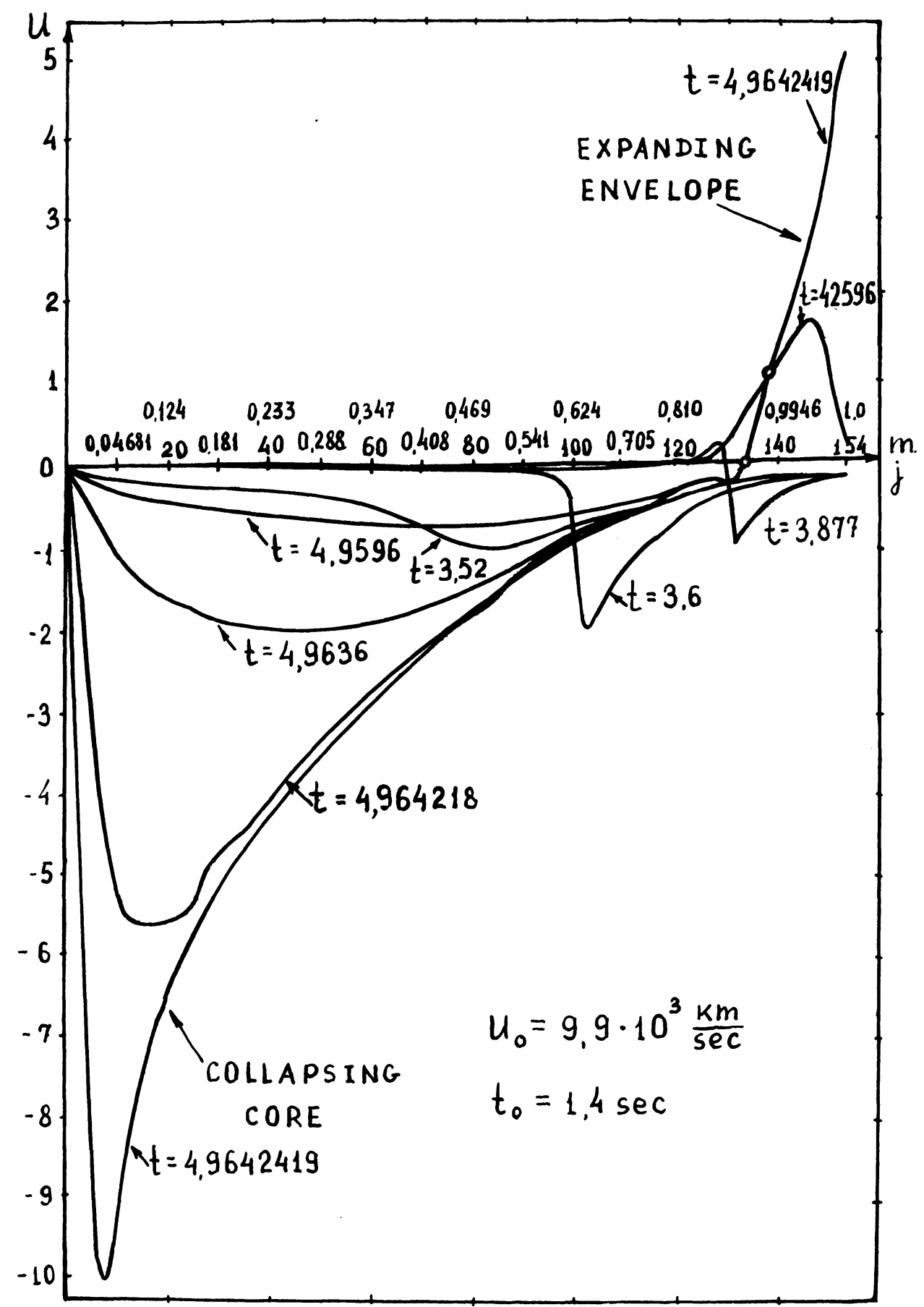

Fig. 8. Distributions of velocity in the star of $10 M_{\odot}$ at various moments of development of dynamic instability. The velocity and time units are $u_{0}=9.9 \times 10^{3} \mathrm{~km} \mathrm{~s}^{-1}, t_{0}=1.4 \mathrm{~s}$. The collapsing core and expanding envelope may be seen at the same time. 
different moments of time. It should be noted that we have a collapsing core and an expanding envelope at the same time.

\section{Theoretical Light Curves of Supernovae}

Figures 9-10 shows theoretical light curves of the compact and expanded models, and

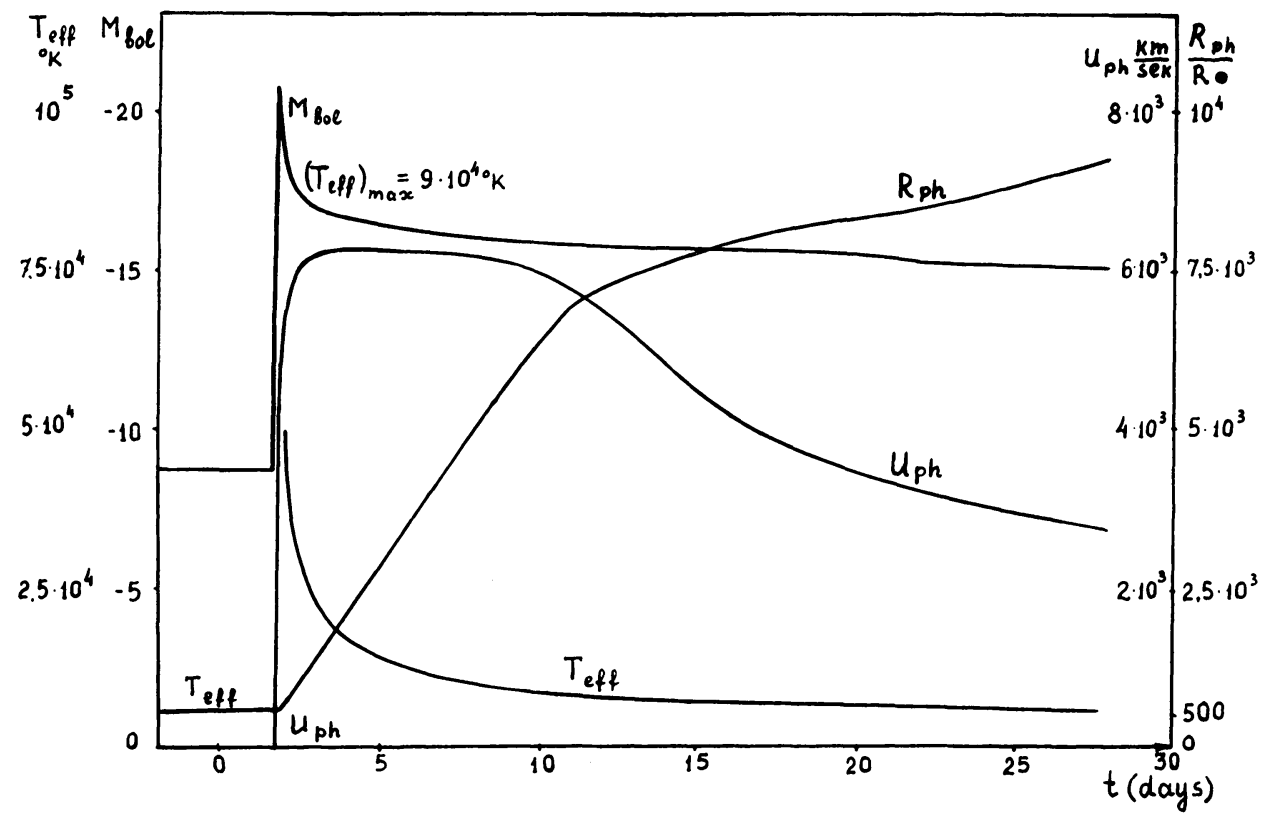

Fig. 9. Theoretical light curve and characteristics of supernova photosphere for presupernova with $R=500 R_{\odot}$.

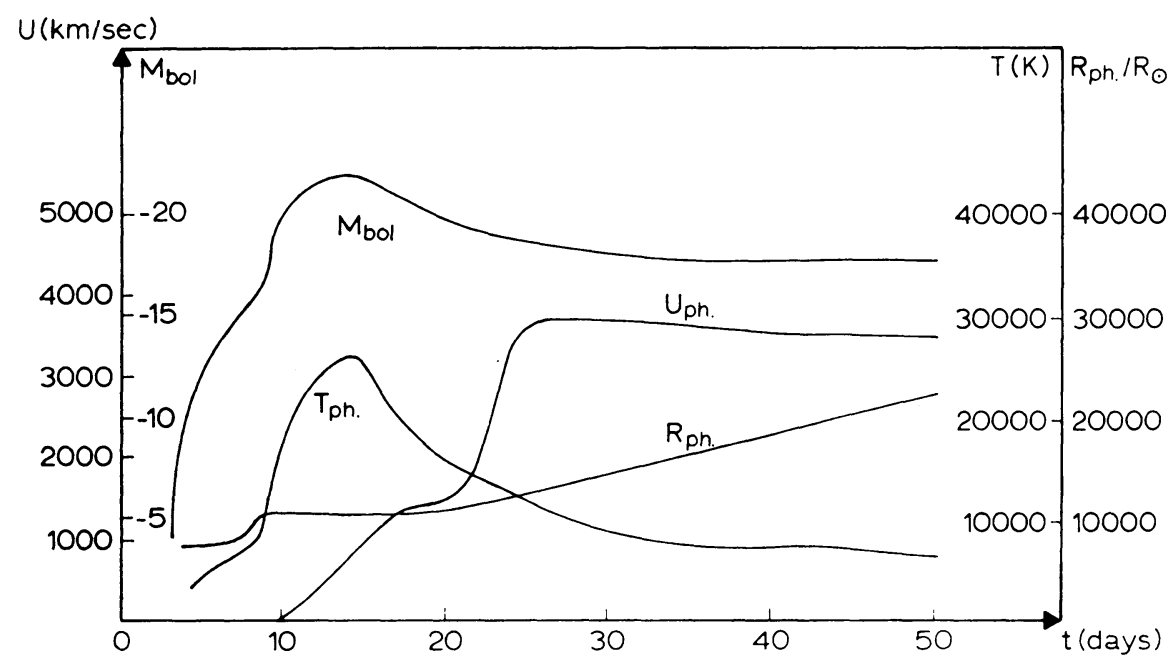

Fig. 10. The same as in Figure 9 but for presupernova with $R=10^{4} R_{\odot}$. 
also characteristics of the photosphere such as radius, effective temperature and the speed of matter emerging through the photosphere. From the comparison of observed light curves with theoretical ones (Figure 11), it follows that theoretical and observed light curves are characterized by the same parameters. However, there is some discrepancy basically involving in the shape of light curves. One should think here of theoretical results having been derived from the very crude presupernova models. For example it was assumed, for models with extended envelopes, that the density of the envelope falls off with the cube of the distance and is broken off at the surface of the star. We believe that the fitting between the theory and observations may be improved by a suitable selection of the law of density variation.

The lack of hydrostatic equilibrium of the extended envelopes has not practically any influence on the theoretical light curves since the speed of ejected matter is con-

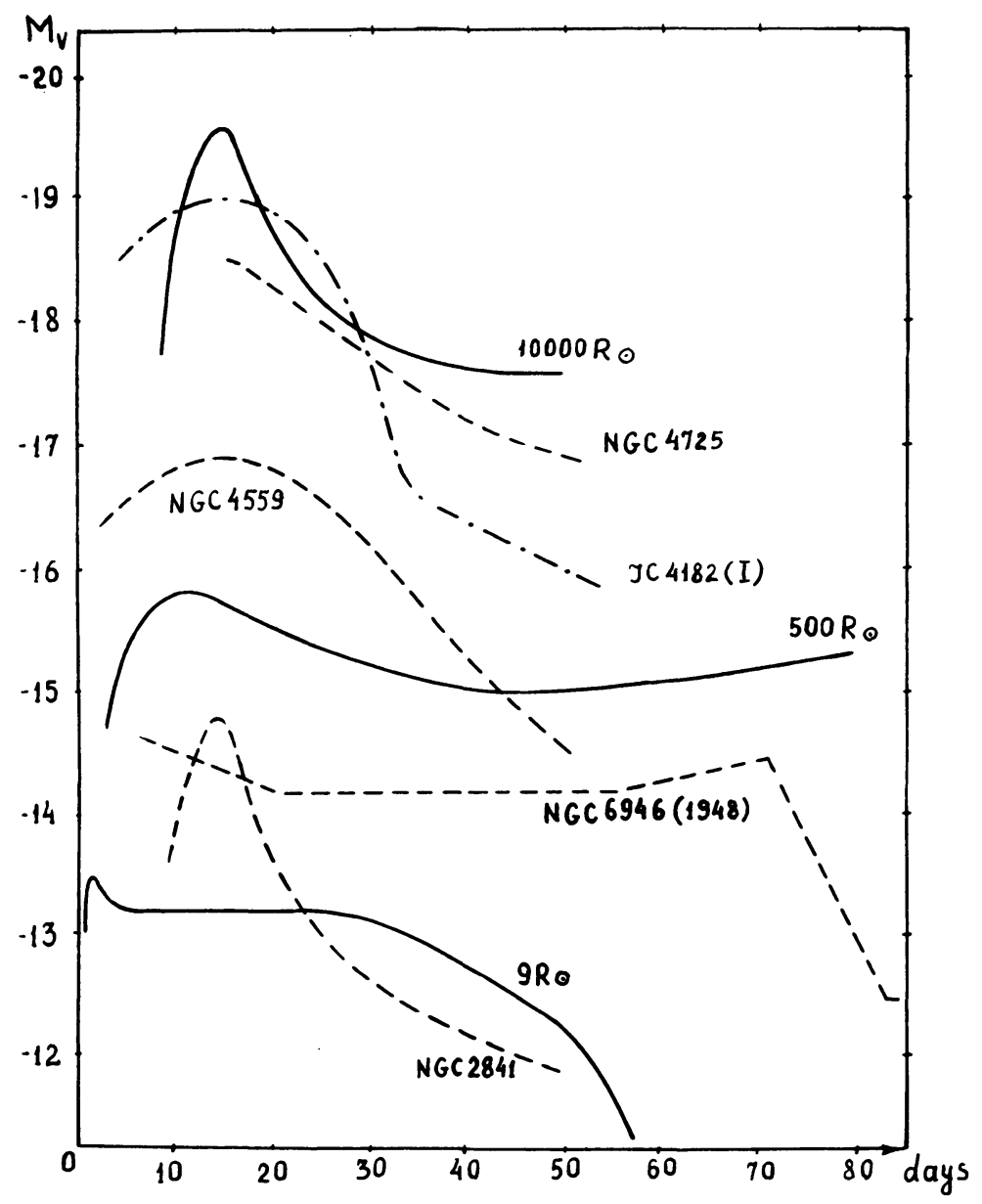

Fig. 11. Comparison of theoretical light curves (continuous curves) with observations (dotted curves). 
siderably greater than the speed of sound. The extended envelope may be formed as a result of mass loss in the late stages of stellar evolution. For the stationary mass loss the density at great distance from the star is in inverse square proportion to the distance:

$$
\varrho=\frac{|\dot{M}|}{4 \pi v r^{2}},
$$

where $\dot{M}$ denotes the rate of mass loss; and $v$, the velocity of ejection. The position of the photosphere $R_{\mathrm{ph}}$ is determined by the relation:

$$
\tau\left(R_{\mathrm{ph}}\right)=\int_{R_{\mathrm{ph}}}^{\infty} \varrho \varkappa \mathrm{d} r=\frac{2}{3} .
$$

After substituting (12) to (13) and integrating one finds

$$
|\dot{M}|=10^{-9} \frac{\bar{v}}{\bar{x}} R_{\mathrm{ph}}\left(M_{\odot} \mathrm{yr}^{-1}\right),
$$

where $\bar{x}$ and $\bar{v}$ are some means of the opacity and the velocity; $\bar{v}$ is in $\mathrm{km} \mathrm{s}^{-1}$, and $R_{\mathrm{ph}}$, in $R_{\odot}$. The velocity of ejection is of the order of $10-100 \mathrm{~km} \mathrm{~s}^{-1}$. According to (14) with $\bar{\varkappa}=0.4 \mathrm{~cm}^{2} \mathrm{~g}^{-1}$ the rate of mass loss of the order of

$$
|\dot{M}|=2.5 \times\left(10^{-4}-10^{-3}\right) M_{\odot} \mathrm{yr}^{-1}
$$

is necessary that the radius of photosphere should be as large as $10^{4} R_{\odot}$. Thus, a quite moderate loss of mass is needed for producing the extended atmosphere. It should be noted that the latter may be cold and transparent immediately before the supernova outburst. It was just assumed in the above calculations. From the theoretical point of view it is only important that the dimensions of the photosphere should be of the order of $10^{4} R_{\odot}$ after the sharp increase of opacity due to heating of the matter by the thermal wave.

Hence, presupernova may not surely look like a red (or infrared) supergiant. It may be a star with intermediate effective temperature (of spectral type $G$ or F), embedded in an extended, cold, and transparent envelope.

\section{References}

Arnett, W. D.: 1966, Can. J. Phys. 44, 2553.

Arnett, W. D.: 1967, Can. J. Phys. 45, 1621.

Arnett, W. D.: 1969, Astrophys. Space Sci. 5, 180.

Barkat, Z., Buchler, J. R., and Wheeler, J. C.: 1970, Astrophys. Letters 6, 117.

Bisnovatyi-Kogan, G. S. and Kazhdan, Ya. M.: 1966, Astron. Zh. 43, 761.

Bisnovatyi-Kogan, G. S. and Seidov, Z. F.: 1969, Astrofizika 5, 243.

Bisnovatyi-Kogan, G. S. and Seidov, Z. F.: 1970, Astron. Zh. 47, 139.

Bisnovatyi-Kogan, G. S.: 1970, Astron. Zh. 47, 813.

Bruenn, S. W.: 1971, Astrophys. J. 168, 203.

Bruenn, S. W.: 1972, Astrophys. J. Suppl. 24, 283.

Buchler, J. R., Wheeler, J. C., and Barkat. Z.: 1971, Astrophys. J. 167, 465. 
Chechetkin, V. M.: 1969, Astron. Zh. 46, 202 and 206.

Colgate, S. A. and White, R.: 1966, Astrophys. J. 143, 626.

Colgate, S. A. and McKee, Ch.: 1969, Astrophys. J. 157, 623.

Colgate, S. A.: 1971, Astrophys. J. 163, 221.

Domogatsky, G. V.: 1969, Nauch. Inform. Astron. Soviet. Acad. Sci. USSR 13, 94.

Ergma, E. and Paczyński, B.: 1973, in preparation.

Finzi, A. and Wolf, R. A.: 1967, Astrophys. J. 150, 115.

Fowler, W. and Hoyle, F.: 1965, Neutrino Processes and Pair Formation in Massive stars and Supernovae, University of Chicago Press, Chicago-London.

Fraley, G.: 1968, Astrophys. Space Sci. 2, 96.

Grassberg, E. K. and Nadyozhin, D. K.: 1969a, Astron. Zh. 46, 745.

Grassberg, E. K. and Nadyozhin, D. K.: 1969b, Nauch. Inform. Astron. Soviet. Acad. Sci. USSR 13, 96.

Grassberg, E. K., Imshennik, V. S., and Nadyozhin, D. K.: 1971, Astrophys. Space Sci. 10, 28.

Hansen, C. J. and Wheeler, J. C.: 1969, Astrophys. Space Sci. 3, 464.

Ikeuchi, S., Nakazawa, K., Murai, T., Hoshi, R., and Hayashi, C.: 1971, Prog. Theor. Phys. Japan 46, 1713.

Ikeuchi, S., Nakazawa, K., Murai, T., Hoshi, R., and Hayashi, C.: 1972, Prog. Theor. Phys. Japan 48, 1870.

Imshennik, V. S. and Nadyozhin, D. K.: 1964, Astron. Zh. 41, 829.

Imshennik, V. S. and Nadyozhin, D. K.: 1965, Astron. Zh. 42, 1954.

Imshennik, V. S. and Nadyozhin, D. K.: 1967, in Ya. B. Zel'dovich and I. D. Novikov (eds.). Relativistic Astrophysics, Nauka, Moscow.

Imshennik, V. S. and Nadyozhin, D. K.: 1972, JETP 63, 1548.

Imshennik, V. S., Nadyozhin, D. K., and Pinaev, V. S.: 1966, Astron. Zh. 43, 1215.

Imshennik, V. S., Nadyozhin, D. K., and Pinaev, V. S.: 1967, Astron. Zh. 44, 768.

Imshennik, V. S. and Morozov, Yu. I.: 1969, Astron. Zh. 46, 800.

Imshennik, V. S. and Chechetkin, V. M.: 1970, Astron. Zh. 47, 929.

Ivanova, L. N., Imshennik, V. S., and Nadyozhin, D. K.: 1967, preprint IAM Acad. Sci. USSR.

Ivanova, L. N., Imshennik, V. S., and Nadyozhin, D. K.: 1969, Nauch. Inform. Astron. Soviet. Acad. Sci. USSR 13, 3.

Ivanova, L. N., Imshennik, V. S., and Chechetkin, V. M.: 1973, in preparation.

LeBlanc, J. M. and Wilson, J. R.: 1970, Astrophys. J. 161, 541.

Morrison, P. and Sartori, L.: 1969, Astrophys. J. 158, 541.

Mustel, E. R.: 1971, Astron. Zh. 48, 3.

Nadyozhin, D. K. and Chechetkin, V. M.: 1969, Astron. Zh. 46, 270.

Paczyński, B.: 1970, Acta Astron. $20,47$.

Paczyński, B.: 1971, Preprint 1, Warsaw, Polish Acad. Sci.

Paczyński, B.: 1972, Astrophys. Letters 11, 53.

Poveda, A. and Woltjer, L.: 1968, Astron. J. 73, 65.

Rakavy, G. and Shaviv, G.: 1967, Astrophys. J. 148, 803.

Rose, W. K.: 1969, Astrophys. J. 155, 491.

Tsuruta, S. and Cameron, A. G. W.: 1970, Astrophys. Space Sci. 7, 374.

Uus, U.: 1970a, Nauch. Inform. Astron. Soviet. Acad. Sci. USSR 17, 3.

Uus, U.: 1970b, Nauch. Inform. Astron. Soviet. Acad. Sci. USSR 17, 25.

Varshavsky, V. I. and Tutikov, A. V.: 1972, Nauch. Inform. Astron. Soviet. Acad. Sci. USSR 23, 47. Varshavsky, V. I. and Tutukov, A. V.: 1973, Nauch. Inform. Astron. Soviet. Acad. Sci. USSR 26, 35.

Zel'dovich, Ya. B. and Novikov, I. D.: 1971, K. S. Thorne and W. D. Arnett (eds.), Relativistic Astrophysics, Vol. 1: Stars and Relativity, The University of Chicago Press, Chicago and London. 